\title{
Risks of accidental exposure to blood in Tunisian intensive care units
}

\author{
Nakhli M.S. ${ }^{1}$, Kahloul M. ${ }^{1}$, Kacem I. ${ }^{2}$, Guedri S. ${ }^{2}$, Mhamdi S. ${ }^{1}$, Mrizak N. ${ }^{2}$ \\ ${ }^{1}$ Sahloul Hospital, Dept of Anaesthesiology \& Intensive Care, Sousse, Tunisia, \\ ${ }^{2}$ Farhat Hached Hospital, Department of Occupational Medicine, Sousse, Tunisia
}

\section{Introduction}

Accidental Blood exposure (ABE) is an important occupational hazard among physicians. They are, therefore, at risk of occupational infections. The major risk of $A B E$ is due to blood-borne viruses such as Human Immunodeficiency Virus (HIV), Hepatitis B (HBV) and Hepatitis C (HCV). The intensive care unit activity is considered to be at high risk of ABE. Indeed, the rapid turnover of staff members, and subsequently sometimes their lack of experience, emergency situations, and the wide diversity of care procedures performed are important factors contributing to the increased risk of this incident.

This study aims to determine the prevalence and the characteristics of ABE in Tunisian intensive care units.

\section{Methods}

This is a retrospective study analyzing all the cases of $A B E$, occurred in intensive care units and declared in the department of Occupational medicine of the teaching Hospital Farhat Hached of Sousse during the year of 2015. The data collection was based on the data sheets of the ABE declarations used in the occupational department and inspired from those drawn up by the South-East CCLIN in France.

\section{$\underline{\text { Results }}$}

Overall, 109 cases of $A B E$ were declared, of which 46 occurred in the intensive care units $(42,2 \%)$.

The mean age of the study population was $34.4 \pm 10.7$ years with a sex ratio of 0.27 .

The victims were essentially paramedics ( $43.4 \%$ of cases) (Figure 1$)$. The accident was secondary to a needle stick in $84.8 \%$ of cases (Figure 2 ).

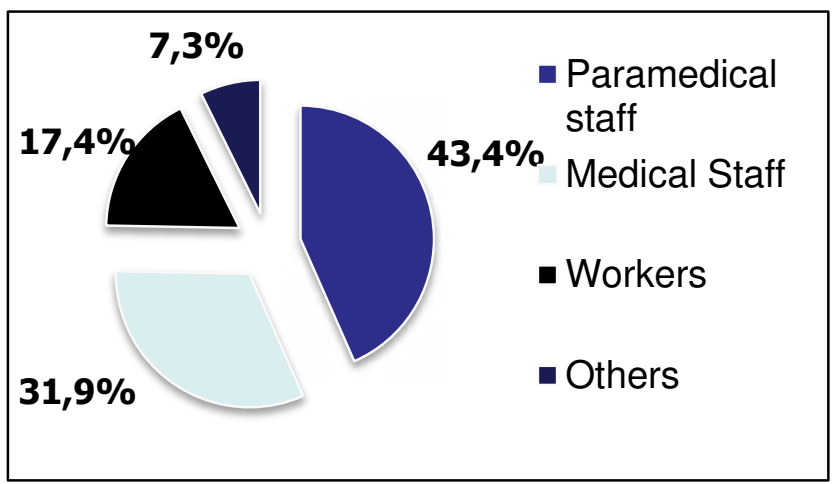

Figure 1 : Distribution of the population by occupational category

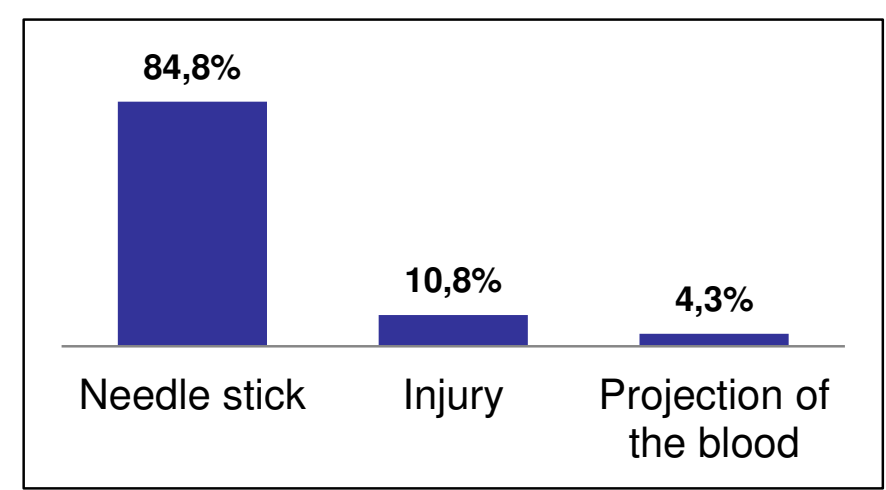

Figure 2 : Mechanism of accident
Of the $A B E$ victims, $67,3 \%$ were adequately vaccinated against hepatitis $B$ (Figure 3 ) but the HBS antibody was not performed in any case.

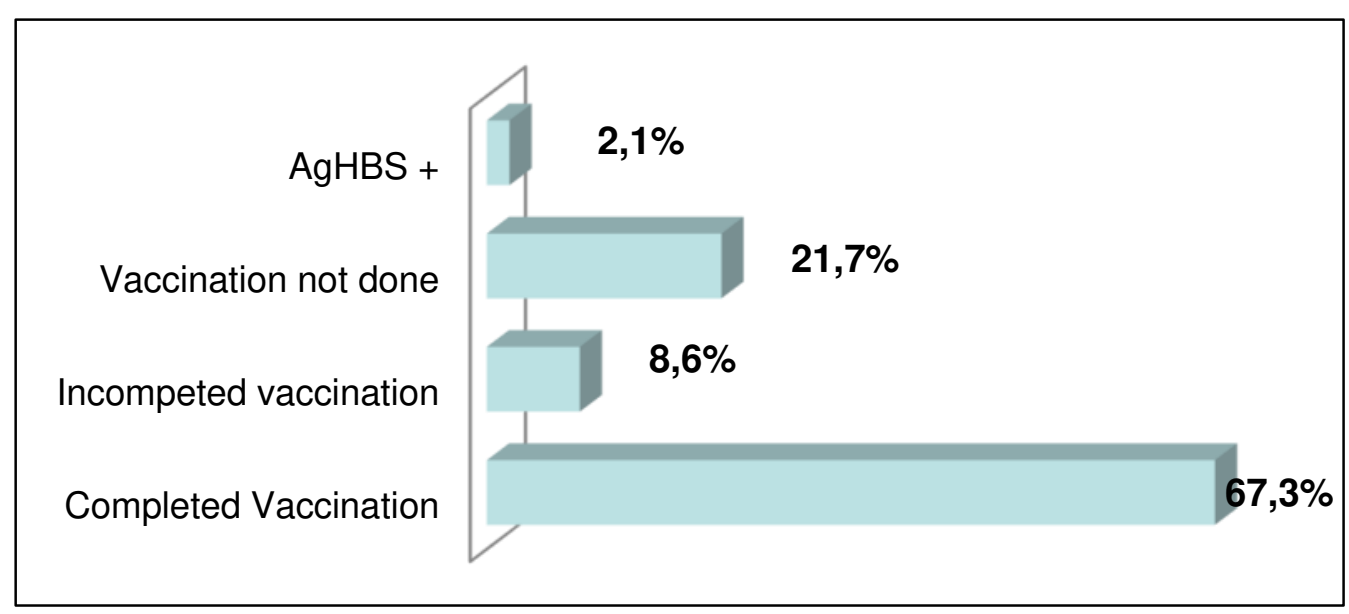

Figure 3 : Victims vaccine status

The wearing of gloves was noted in $32.6 \%$ of the cases. The serological status of the source patient was unknown in $86.9 \%$. Serological surveillance recommended for 18 victims was done only in 4 cases. The immediate management after the ABE was consistent with universal recommendations in $43.4 \%$ of cases. This compliance with the applicable universal recommendations was significantly higher for staff with less than 5 years' seniority $(p<0.05)$.

\section{References}

\section{Discussion}

According to the World Health Organization, accidental blood and fluid exposure accounted for about $40 \%$ of hepatitis B and C infections and $2.5 \%$ of HIV infections in healthcare workers worldwide. Their estimated annual costs for tests and treatments varied from \$6.1 million in France to \$118-591 million in the United States [1] .

As the intensive care unit requires various procedures, it may be at higher risk of $A B E$ than the medical wards. $A$ less working experience, poor work conditions in regard to staffing and resource adequacy can also increase significantly the ABE risk $[2,3]$.

Several leads are possible to improve the acknowledgment of occupational exposures to blood and other body fluids in the intensive care units. Firstly, the use of safety materials seems to be a quick and simple step.

Secondly, it appears essential to create a specific training for residents and young physicians, and to assess its efficiency. This could encourage the declaration of ABE by physicians, and decrease their potential consequences.

Thirdly, the major obstacle to the declaration is the complexity and the length of the procedure. So, training a specific manager of $A B E$ in each medical department can accelerate the procedure and guarantee a personalized approach [2].

\section{Conclusion}

Education, training, the strict application of standard/universal procedures (protective gowns, gloves, masks, glasses, etc...), and also the implementation of local standardized procedures are of paramount importance to reduce $A B E$ incidence. 